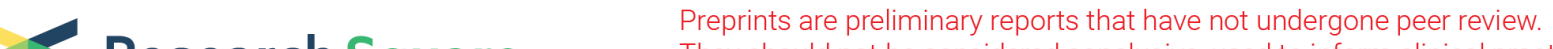 $\begin{array}{ll}\text { Research Square } & \text { They should not be considered conclusive, used to inform clinical practice, } \\ \text { or referenced by the media as validated information. }\end{array}$
}

\section{Left Atrial Appendage Circular RNAs Are New Predictors of Atrial Fibrillation Recurrence After Surgical Ablation in Valvular Atrial Fibrillation Patients: a Case Control Study}

\section{Xiyu Zhu}

Department of Cardio-Thoracic Surgery, Nanjing Drum Tower Hospital, The Affiliated Hospital of Nanjing University Medical School

\section{Yali Wang}

Department of Cardio-Thoracic Surgery, Nanjing Drum Tower Hospital, The Affiliated Hospital of Nanjing University Medical School

\section{Ran Mo}

Department of Burn \& Plastic Surgery, Nanjing Drum Tower Hospital, The Affiliated Hospital of Nanjing University Medical School

\section{Hoshun Chong}

Department of Cardio-Thoracic Surgery, Nanjing Drum Tower Hospital, The Affiliated Hospital of Nanjing University Medical School

\section{Hailong Cao}

Department of Cardio-Thoracic Surgery, Nanjing Drum Tower Hospital, The Affiliated Hospital of Nanjing University Medical School

\section{Fudong Fan}

Department of Cardio-Thoracic Surgery, Nanjing Drum Tower Hospital, The Affiliated Hospital of Nanjing University Medical School

\section{Dongjin Wang}

Department of Cardio-Thoracic Surgery, Nanjing Drum Tower Hospital, The Affiliated Hospital of Nanjing University Medical School

\section{Qing Zhou ( zhouqing@njglyy.com )}

Department of Cardio-Thoracic Surgery, Nanjing Drum Tower Hospital, The Affiliated Hospital of Nanjing University Medical School

\section{Research Article}

Keywords: atrial fibrillation recurrence, surgical ablation, circular RNA, predictive model

Posted Date: February 25th, 2021 
DOl: https://doi.org/10.21203/rs.3.rs-238970/v1

License: (c) (1) This work is licensed under a Creative Commons Attribution 4.0 International License. Read Full License 


\section{Abstract}

Background: Atrial fibrillation recurrence after surgical ablation in valvular atrial fibrillation patients will increase the mortality and morbidity during follow-up. In this study, we attempted to evaluate the relationship between circular RNAs in left atrial appendage and atrial fibrillation recurrence to establish a predictive model for early intervention.

Methods: A retrospective case control study of all patients who underwent surgical ablation during valve surgery was performed. The expression level of circular RNAs were detected in the left atrial appendage tissue after surgery. 24 hours Holter examination was used to evaluate the status of heart rhythm during follow-up. The independent risk factors of atrial fibrillation recurrence were analyzed by multivariate analysis. The predictive model of atrial fibrillation recurrence was visualized by Nomogram and was tested by receiver operating characteristic curve analysis. Kaplan-Meier analysis was performed to compare the rate of freedom from atrial fibrillation recurrence after surgery.

Results: A total of 136 patients completed the research strategy from September 2018 to June 2019, 81 (59.6\%) patients restored sinus rhythm and $55(40.4 \%)$ patients experienced atrial fibrillation recurrence. Increased age $(P=0.002)$, longer $A F$ duration $(P<0.001)$ and increased expression of circ 81906-RYR2 $(P<0.001)$, circ 44782-LAMA2 $(P=0.002)$, circ 418-KCNN2 $(P=0.011)$ and circ 35880-ANO5 $(P<0.001)$ were detected in atrial fibrillation recurrence group. Multivariate analysis revealed that increased age (odd ratio=1.072, $\mathrm{P}=0.006$ ), longer $\mathrm{AF}$ duration (odd ratio=1.007, $\mathrm{P}=0.036$ ) and increased expression of circ 81906- $R$ YR2 (odd ratio=2.210, $\mathrm{P}<0.001$ ) were independent risk factors for recurrence. The area under the curve was 0.77 and the cut-off value was 70 points of the predictive model. The results of Kaplan-Meier plots showed that patients over 70 points were more likely to suffer from atrial fibrillation recurrence during the follow-up.

Conclusions: Circ 81906-RYR2 could be a new predictor of atrial fibrillation recurrence after surgical ablation. A predictive model consists of age, atrial fibrillation duration and expression of circ 81906-RYR2 was alternative for early intervention of atrial fibrillation recurrence.

Trial registration: Retrospectively registered.

\section{Background}

Surgical ablation combined with valve surgery is an effective method to maintain sinus rhythm (SR) during follow-up in valvular atrial fibrillation (AF) patients [1]. Restoration of sinus rhythm is helpful to decrease the incidence of heart failure, re-hospitalization and thrombotic events after surgery [2]. However, the restoration rate after surgery varied among different centers (65\%-95\%) [3-6]. A real-world nationwide study also showed the rate of AF recurrence range from $31-45 \%$ [7]. The outcome of patients with persistent or permanent AF was worse than patients with paroxysmal AF. Several risk factors have been identified in the former studies, such as advanced age, concomitant cardiovascular disease and the length of AF duration $[8,9]$ to predict the possibility of post-operative AF recurrence. It is helpful to find 
some new biomarkers to predict AF recurrence after surgical ablation in AF patients with valvular heart disease (VHD) and make the early therapeutic intervention for those who suffered from AF recurrence. Circular RNA (CircRNA) is a kind of non-coding RNA and which was first described in the early 1990s [10]. Basic researches have revealed that circRNAs can be used as clinical predictors for central nervous disease [11] and cancers [12], our former study have found serval differentially expressed circRNAs in AF patients with VHD [13], however whether these circRNAs can be used as clinical biomarkers for outcome of surgical ablation still need further evaluation. The aim of this study was to evaluate the prognostic value of circRNAs for the outcome of surgical ablation in persistent AF patients with VHD. We present the following article in accordance with the STROBE reporting checklist.

\section{Methods}

\section{Patients enrollments}

A total of 150 persistent AF patients with VHD were continuously enrolled in this study from September 2018 to June 2019. Preoperative transthoracic echocardiography (TTE) was performed to evaluate the subtype of valve diseases, the degree of valve diseases, left atrial diameter (LAD) and the left ventricular ejection fraction (LVEF). Persistent AF was diagnosed by the preoperative 24 hours Holter examination in patients with continuous AF over 3 months. Patients over 50 years old need to finish coronary angiography to evaluate the coronary artery lesion. Demographic data (including age, gender and body mass index), cardiovascular risk factors (hypertension, diabetes mellitus, chronic kidney disease, smoke or alcohol abuse), concomitant diseases, results of TTE and intro-operative data (operation time, cardiopulmonary bypass time, aortic crossclamp time, valve repair or replacement and type of artificial valve) were recorded for further exploration. The exclusion criteria were listed as follow: 1) age of patients was less than 18 or more than 75 years old, 2) patients with aortic diseases, infective endocarditis, congenital heart diseases or acute myocardial infraction, 3) non-valvular AF, 4) patients died during perioperative period. Remote ECG monitoring was settled for all patients in the first 5 days after operation. Beta blocker or amiodarone was used to maintain the heart rate between 60 and $70 \mathrm{bpm}$. All patients discharged without complications and received more than 1 year follow up. Telephone interviews were performed monthly and all patients required to finish the 24 hours Holter examination during visit to the outpatient clinic 3 months, 6 months and 1 year after operation to evaluate the arrhythmia events during follow-up. Patients were divided into two groups as sinus rhythm maintenance (SRM) and atrial fibrillation recurrence (AFR) according to the results of the 24 hours Holter in 3 months and 1 year separately. Patients who experienced AF events in 3 months received at least once cardioversion and following antiarrhythmic drugs therapy.

\section{Surgical procedures}

Surgical ablation also known as Cox-Maze IV procedure [14] was performed in all patients. Cardioblate surgical ablation system (Medtronic, Minneapoils, USA) was selected to finish the radiofrequency ablation. Bi-atrial ablations were achieved, left atrial appendage (LAA) and Marshall ligament were 
resected during the operation. Valve replacement or repair were performed in patients with valve diseases. Coronary artery bypass graft was operated in patients with coronary artery lesions. In brief, after cardiopulmonary bypass (CPB) establishment, pulmonary vein isolation and the ablation of right atrium was performed before ascending aorta clamping, then the ablation of left atrium was performed according to the procedure of Cox-Maze IV. The ablation energy was settled range from 25 Walt to 30 Walt. There were 40 mitral valve repair, 10 aortic valve repair, 125 tricuspid valve repair, 66 mitral valve replacement, 26 aortic valve replacement, 1 tricuspid valve replacement and 13 coronary artery bypass graft performed as the concurrent surgery.

\section{Examination of circRNAs in LAA tissue}

LAA was resected in all patients enrolled in this study. The specimen was divided into two parts after resection, of which, one was immediately frozen into liquid nitrogen for RNA isolation and further experiments, the other was prepared for pathological analysis.

The expression level of circRNAs in LAA were detected by using quantitative real-time reverse transcription polymerase chain reaction (qRT-PCR). Total RNA was extracted from LAA sample using TRIzol Reagent (Cat. No 15596018, ThermoFisher Scientific) according to the manufacturer. The concentration of total RNA was measured by NanoDrop One (ThermoFisher Scientific) and then it was reverse transcribed to synthesize cDNA using HiScript II Q Select RT SuperMix for qRT-PCR (Cat. No R23201, Vazyme). The expression of specific circRNA (circ 81906-RYR2, circ 44782-LAMA2, circ 418-KCNN2, circ 35880-ANO5 and circ 255-ITGA7) was measured by qRT-PCR with ChamQ Universal SYBR qPCR Master Mix (Cat. No Q711-02, Vazyme) and LightCycler 480 Instrument II (Roche Molecular System, Inc). $G A P D H$ was selected as the internal reference and the relative expression level of circRNA was calculated with the $2^{-\triangle \triangle C T}$ method. The primers used in this study were listed in Supplementary Table 1.

\section{Statistical analysis}

Numerical variables were described as mean \pm standard deviation (SD), categorical variables were described as case numbers. Statistical analysis was performed by using SPSS version 23.0. Differences of continuous variables between groups were measured by Student's $t$ test or Mann-Whitney Utest. Chisquare test was used to analyze the categorical variables, fisher's exact was used when sample size was less than five. Kaplan-Meier analysis was used to compare the rate of freedom from AF. Multivariate logistic regression analysis was performed to find the predictors of AF recurrence. Rms package was used to visualize the predictive model and nomogramFormula package was used to calculate the total score of each patient by using the $\mathrm{R}$ language (version 4.0.3). Receiver operating characteristic (ROC) curve was used to evaluate the sensitivity and specificity of the prediction model. Youden's index was calculated by the formula $J=$ sensitivity + specificity -1 . Kaplan-Meier analysis was performed to compare the rate of freedom from AF recurrence after surgery. $P$ value less than 0.05 was considered significantly different.

\section{Results}


150 persistent AF patients with VHD were continuously enrolled in this study from September 2018 to June 2019. According to the exclusion criteria, seven patients were excluded from this study. Four patients were due to overage, two patients were excluded because of the diagnosis of aortic disease, another one patient was excluded because the valve disease was aroused by acute myocardial infraction. The remained 143 patients were put forward to receive the surgical ablation procedure during operation. There were seven cases lost follow-up. Finally, data of 136 patients were remained for further analysis. The mean follow-up period was $19.8 \pm 2.9$ months. The clinical characters were listed in Tables 1 and 2 . 
Table 1

Clinical characteristics of valvular PeAF patients in 3 months follow-up

\begin{tabular}{|c|c|c|c|}
\hline & SRM1 & AFR1 & $P$ value \\
\hline & $N=72$ & $N=64$ & \\
\hline Age (year) & $58.9 \pm 9.6$ & $61.4 \pm 8.0$ & 0.098 \\
\hline Gender (Male/Female) & $36 / 36$ & $29 / 35$ & 0.610 \\
\hline $\mathrm{BMI}\left(\mathrm{kg} / \mathrm{m}^{2}\right)$ & $23.3 \pm 3.6$ & $24.4 \pm 3.3$ & 0.055 \\
\hline AF duration (month) & $41.0 \pm 60.6$ & $61.2 \pm 77.6$ & 0.023 \\
\hline \multicolumn{4}{|l|}{ Cardiovascular risk factors } \\
\hline Hypertension (n/\%) & $22(30.6 \%)$ & $25(39.1 \%)$ & 0.367 \\
\hline Diabetes mellitus (n/\%) & $3(4.2 \%)$ & $5(7.8 \%)$ & 0.475 \\
\hline CKD (n/\%) & $1(1.4 \%)$ & $2(3.1 \%)$ & 0.601 \\
\hline Smoke abuse (n/\%) & $7(9.7 \%)$ & $5(7.8 \%)$ & 0.769 \\
\hline Alcohol abuse (n/\%) & $5(6.9 \%)$ & $2(3.1 \%)$ & 0.447 \\
\hline Coronary artery disease (n/\%) & $10(13.9 \%)$ & $11(17.2 \%)$ & 0.640 \\
\hline Stroke (n/\%) & $4(5.6 \%)$ & $3(4.7 \%)$ & 1.000 \\
\hline \multicolumn{4}{|l|}{ Transthoracic echocardiography } \\
\hline LAD (cm) & $5.1 \pm 0.9$ & $5.5 \pm 1.1$ & 0.024 \\
\hline EF (\%) & $52.4 \pm 6.9$ & $53.0 \pm 7.8$ & 0.403 \\
\hline Rheumatic valvular disease $(\mathrm{n} / \%)$ & $40(55.6 \%)$ & $32(50.0 \%)$ & 0.606 \\
\hline \multicolumn{4}{|l|}{ NYHA Class } \\
\hline Class I & 1 & 4 & \multirow[t]{4}{*}{0.343} \\
\hline Class II & 34 & 24 & \\
\hline Class III & 36 & 34 & \\
\hline Class IV & 1 & 2 & \\
\hline $\mathrm{BNP}(\mathrm{pg} / \mathrm{mL})$ & $345.5 \pm 301.7$ & $319.2 \pm 301.3$ & 0.769 \\
\hline CRP (mg/L) & $7.2 \pm 20.9$ & $8.2 \pm 15.5$ & 0.346 \\
\hline
\end{tabular}

Abbreviations: $B M I$, body mass index; $C K D$, chronic kidney disease; $L A D$, left atria diameter; $E F$, ejection fraction; BNP, brain natriuretic peptide; CRP, C-reactive protein; eGFR, estimated glomerular filtration rate 


\begin{tabular}{|c|c|c|c|}
\hline & \multirow{2}{*}{$\begin{array}{l}\text { SRM1 } \\
\mathrm{N}=72\end{array}$} & \multirow{2}{*}{$\begin{array}{l}\text { AFR1 } \\
N=64\end{array}$} & \multirow[t]{2}{*}{$P$ value } \\
\hline & & & \\
\hline $\operatorname{eGFR}\left(\mathrm{mL} /\left(\mathrm{min}^{\star} 1.73 \mathrm{~m}^{2}\right)\right)$ & $93.5 \pm 23.7$ & $95.1 \pm 21.6$ & 0.675 \\
\hline \multicolumn{4}{|c|}{$\begin{array}{l}\text { Abbreviations: BMI, body mass index; CKD, chronic kidney disease; LAD, left atria diameter; EF, } \\
\text { ejection fraction; BNP, brain natriuretic peptide; CRP, C-reactive protein; eGFR, estimated glomerular } \\
\text { filtration rate }\end{array}$} \\
\hline
\end{tabular}


Table 2

Clinical characteristics of valvular PeAF patients in one-year follow-up

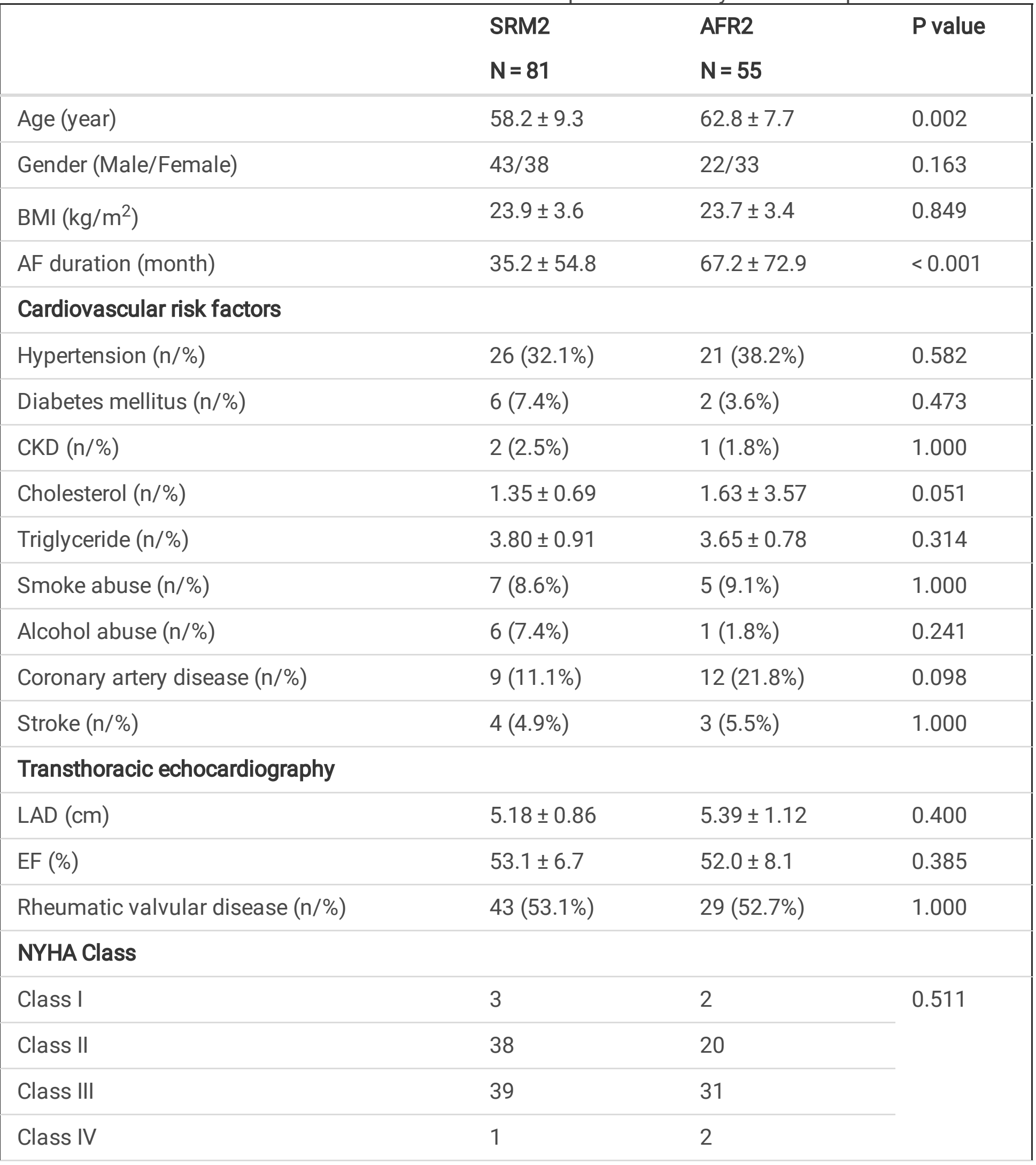

Abbreviations: $B M I$, body mass index; CKD, chronic kidney disease; $L A D$, left atria diameter; $E F$, ejection fraction; BNP, brain natriuretic peptide; CRP, C-reactive protein; eGFR, estimated glomerular filtration rate; $\mathrm{CPB}$, cardiopulmonary bypass time; $\mathrm{ACC}$, aortic crossclamp; $\mathrm{CABG}$, coronary artery bypass graft 


\begin{tabular}{|c|c|c|c|}
\hline & SRM2 & AFR2 & \multirow[t]{2}{*}{$P$ value } \\
\hline & $N=81$ & $N=55$ & \\
\hline BNP (pg/mL) & $316.5 \pm 264.0$ & $357.2 \pm 347.8$ & 0.980 \\
\hline CRP (mg/L) & $7.26 \pm 20.08$ & $8.24 \pm 16.10$ & 0.304 \\
\hline $\operatorname{eGFR}\left(\mathrm{mL} /\left(\min * 1.73 \mathrm{~m}^{2}\right)\right)$ & $93.2 \pm 22.8$ & $96.1 \pm 22.5$ & 0.558 \\
\hline \multicolumn{4}{|l|}{ Intraoperative data } \\
\hline Operation time (hour) & $4.40 \pm 0.99$ & $4.30 \pm 0.98$ & 0.610 \\
\hline CPB time (min) & $153.0 \pm 44.2$ & $144.7 \pm 41.5$ & 0.242 \\
\hline ACC time (min) & $117.4 \pm 43.3$ & $109.2 \pm 40.3$ & 0.235 \\
\hline Mitral valve repair (n) & 23 & 17 & \multirow[t]{2}{*}{0.691} \\
\hline Mitral valve replacement (n) & 35 & 31 & \\
\hline Aortic valve repair (n) & 7 & 3 & \multirow[t]{2}{*}{1.000} \\
\hline Aortic valve replacement (n) & 17 & 10 & \\
\hline Tricuspid valve repair (n) & 74 & 51 & \multirow[t]{2}{*}{1.000} \\
\hline Tricuspid valve replacement (n) & 1 & 0 & \\
\hline CABG (n) & 7 & 5 & 1.000 \\
\hline Bioprosthetic valve & 37 & 27 & \multirow[t]{2}{*}{0.107} \\
\hline Mechanical valve & 23 & 7 & \\
\hline \multicolumn{4}{|l|}{ Types of arrhythmia } \\
\hline Persistent atrial fibrillation (n/\%) & & \multicolumn{2}{|l|}{$41(74.5 \%)$} \\
\hline Paroxysmal atrial fibrillation (n/\%) & & \multicolumn{2}{|l|}{$6(10.9 \%)$} \\
\hline Atrial flutter (n/\%) & & \multicolumn{2}{|l|}{$8(14.5 \%)$} \\
\hline Total score > 70 points (n/\%) & $26(32.1 \%)$ & $40(72.7 \%)$ & $<0.001$ \\
\hline \multicolumn{4}{|c|}{$\begin{array}{l}\text { Abbreviations: BMI, body mass index; CKD, chronic kidney disease; } L A D \text {, left atria diameter; } E F \text {, } \\
\text { ejection fraction; BNP, brain natriuretic peptide; CRP, C-reactive protein; eGFR, estimated glomerular } \\
\text { filtration rate; CPB, cardiopulmonary bypass time; ACC, aortic crossclamp; CABG, coronary artery } \\
\text { bypass graft }\end{array}$} \\
\hline
\end{tabular}

Patients were split up into two groups at 3 months or 1 year after operation. There were 64 patients (47.1\%) suffered from recurrent AF (AFR1 group) during the first 3 months follow-up. LAD $(5.1 \pm 0.9$ vs 
$5.5 \pm 1.1, P=0.024)$ and $A F$ duration $(41.0 \pm 60.6$ vs $61.2 \pm 77.6, P=0.023)$ were significantly different between two groups (Table 1 ).

According to the Holter examination in one-year follow-up, there were 81 patients getting a result of SR (SRM2 group), other 55 patients were diagnosed AF (AFR2 group). $74.5 \%$ of the recurrent patients were persistent AF. The preoperative clinical characters showed that patients in SRM2 group was 4.6 years younger ( $58.2 \pm 9.3$ vs $62.8 \pm 7.7, \mathrm{P}=0.002$ ) than AFR2 group. The AF duration was shorter in SRM2 group patients $(35.2 \pm 54.8$ months vs $67.2 \pm 72.9$ months, $P<0.001)$. None difference was found in other variables (Table 2). Compared to the follow-up results of three months, 21 patients in AFR1 group restored sinus rhythm and 12 patients in SRM1 group progressed to persistent AF in one-year examination.

Based on the one-year follow up results, the relative expression of circ 81906-RYR $(0.0160 \pm 0.008$ vs $0.0245 \pm 0.0141, \mathrm{P}<0.001)$, circ 44782-LAMA2 (0.00564 \pm 0.00290 vs $0.00754 \pm 0.00384, \mathrm{P}=0.002)$, circ 418-KCNN2 (0.00760 \pm 0.00475 vs $0.01071 \pm 0.00812, \mathrm{P}=0.011)$ and circ 35880-ANO5 $(0.0242 \pm 0.0111$ vs $0.0341 \pm 0.0178, P<0.001)$ were significantly increased in AFR2 group patients compared with SRM2 group (Fig. 1 and Table 3).

Table 3

Expression of circRNA in left atria appendage

\begin{tabular}{|c|c|c|c|c|c|c|}
\hline & $\begin{array}{l}\text { SRM1 } \\
\mathrm{N}=72\end{array}$ & $\begin{array}{l}\text { AFR1 } \\
N=64\end{array}$ & $\begin{array}{l}P \\
\text { value }\end{array}$ & $\begin{array}{l}\text { SRM2 } \\
\mathrm{N}=\mathbf{8 1}\end{array}$ & $\begin{array}{l}\text { AFR2 } \\
\mathrm{N}=55\end{array}$ & $\begin{array}{l}\mathrm{P} \\
\text { value }\end{array}$ \\
\hline $\begin{array}{l}\text { circ 81906- } \\
\text { RYR2 }\end{array}$ & $\begin{array}{l}0.0171 \pm \\
0.009\end{array}$ & $\begin{array}{l}0.0220 \pm \\
0.0140\end{array}$ & 0.013 & $\begin{array}{l}0.0160 \pm \\
0.008\end{array}$ & $\begin{array}{l}0.0245 \pm \\
0.0141\end{array}$ & $\begin{array}{l}< \\
0.001\end{array}$ \\
\hline $\begin{array}{l}\text { circ } 44782- \\
\text { LAMA2 }\end{array}$ & $\begin{array}{l}0.00595 \pm \\
0.00287\end{array}$ & $\begin{array}{l}0.00662 \pm \\
0.00379\end{array}$ & 0.069 & $\begin{array}{l}0.00564 \pm \\
0.00290\end{array}$ & $\begin{array}{l}0.00754 \pm \\
0.00384\end{array}$ & 0.002 \\
\hline $\begin{array}{l}\text { circ 418- } \\
\text { KCNN2 }\end{array}$ & $\begin{array}{l}0.00976 \pm \\
0.0120\end{array}$ & $\begin{array}{l}0.00923 \pm \\
0.00635\end{array}$ & 0.107 & $\begin{array}{l}0.00760 \pm \\
0.00475\end{array}$ & $\begin{array}{l}0.01071 \pm \\
0.00812\end{array}$ & 0.011 \\
\hline $\begin{array}{l}\text { circ 35880- } \\
\text { AN05 }\end{array}$ & $\begin{array}{l}0.0266 \pm \\
0.0165\end{array}$ & $\begin{array}{l}0.0302 \pm \\
0.0174\end{array}$ & 0.009 & $\begin{array}{l}0.0242 \pm \\
0.0111\end{array}$ & $\begin{array}{l}0.0341 \pm \\
0.0178\end{array}$ & <.001 \\
\hline $\begin{array}{l}\text { circ 255- } \\
\text { ITGA7 }\end{array}$ & $\begin{array}{l}0.00582 \pm \\
0.00358\end{array}$ & $\begin{array}{l}0.00863 \pm \\
0.0133\end{array}$ & 0.881 & $\begin{array}{l}0.00542 \pm \\
0.00353\end{array}$ & $\begin{array}{l}0.00621 \pm \\
0.00399\end{array}$ & 0.230 \\
\hline
\end{tabular}

Data of the one-year follow up were used to evaluate the risk factor of AF recurrence. Univariate analysis demonstrated that the advanced age $(P=0.004)$, longer $A F$ duration $(P=0.007)$, increased expression of circ 81906-RYR2 $(\mathrm{P}<0.001)$, circ 44782-LAMA2 $(\mathrm{P}=0.003)$, circ 418-KCNN2 $(\mathrm{P}=0.013)$ and circ 35880ANO5 $(\mathrm{P}=0.001)$ were risk factors for AF recurrence (Table 4). Multivariate analysis revealed that age (OR $1.07195 \% \mathrm{Cl} 1.019-1.125 \mathrm{P}=0.007)$, AF duration (OR 1.007 95\% $\mathrm{Cl} 1.000-1.013 \mathrm{P}=0.040$ ) and circ 81906-RYR2 (OR $2.21395 \% \mathrm{Cl} 1.428-3.429 \mathrm{P}<0.001$ ) were significantly independent predictors for $\mathrm{AF}$ 
recurrence (Table 4). A nomogram was established to visualize the predictive model from logistics regression analysis (Fig. 2).

Table 4

Univariate and multivariate analysis of risk factors for recurrence of AF after surgical ablation

\begin{tabular}{|lllllll|}
\hline Variables & \multicolumn{2}{l}{ Univariate analysis } & \multicolumn{4}{c|}{ Multivariate analysis } \\
\cline { 2 - 7 } & OR & $\mathbf{9 5 \%}$ Cl & P value & OR & $95 \%$ Cl & P value \\
\hline Age & 1.065 & $1.020-1.112$ & 0.004 & 1.072 & $1.020-1.127$ & 0.006 \\
\hline AF duration & 1.008 & $1.002-1.014$ & 0.007 & 1.007 & $1.000-1.013$ & 0.036 \\
\hline circ 81906-RYR2 & 2.283 & $1.502-3.470$ & $<0.001$ & 2.210 & $1.425-3.427$ & $<0.001$ \\
\hline circ 44782-LAMA2 & 1.772 & $1.207-2.601$ & 0.003 & & & \\
\hline circ 418-KCNN2 & 1.603 & $1.103-2.328$ & 0.013 & & & \\
\hline circ 35880-ANO5 & 1.997 & $1.336-2.983$ & 0.001 & & & \\
\hline
\end{tabular}

Furthermore, ROC curve was used to evaluate the relationship between circ 81906-RYR2 and AF recurrence. The area under the curve (AUC) value of circ 81906-RYR2 was $0.699(95 \% \mathrm{Cl} 0.609-0.789, \mathrm{P}<$ 0.001 ) (Fig. 3A). After combined with two clinical characters, age and AF duration, the AUC value was 0.77 (95\% Cl 0.690-0.849, P<0.001) (Fig. 3B). The Youden's index of the total score of the predictive model was 70 points with sensitivity of $87.3 \%$ and specificity of $55.6 \%$. Clinical data of all patients was regrouped by the Youden's index. Kaplan-Meier analysis demonstrated that patients with total score more than 70 points showed high recurrence rate of $A F$ in one year after surgery $(50 \%$ vs $20 \% \mathrm{P}<0.001)$ (Fig. 4).

\section{Discussion}

Surgical ablation should be recommended in patients with symptomatic AF during valve surgery. LAA excision is suggested to reduce the incidence of AF induced thrombotic events [1]. Surgical techniques improvements were achieved to decrease the postoperative AF recurrence, however, the outcome of surgical ablation is still not satisfactory in AF patients with VHD, especially those with rheumatic valvular diseases [15]. Limited number of studies have reported that AF duration, left atrial size and the severity of mitral regurgitation were independent risk factors for $\mathrm{AF}$ recurrence in valvular $\mathrm{AF}$ patients $[16,17]$.

CircRNA is a kind of non-coding RNA and is derived from the process of alternative splicing of pre-mRNAs $[18,19]$. The 5 'ends and 3 'ends of the spliced RNA act as the upstream splice acceptor and the downstream splice donor and are finally covalently linked to each other which is known as back-splicing. Researchers have demonstrated that circRNAs can act as a microRNA sponge to regulate miRNA function [20], bind with proteins to regulate the interaction between proteins [21] and form peptide to participate 
different biological process [22]. Recent years, circRNAs as clinical biomarkers have been proved in the central nervous disease [11] and cancers [12], however, the function of circRNAs in AF remain poorly understood. In our former study, we have described the expression profile of circRNAs in AF patients with VHD and validated ten differentially expressed circRNAs in patients LAA tissue [13]. To our knowledge, this was the first study to evaluated the relationship between LAA circRNAs and AF recurrence after surgical ablation.

According to our previous study [13], the five most differentially expressed circRNAs were selected in this study. We found the recurrence rate of valvular persistent AF patients was $40.4 \%$ in our center. The recurrence rate was higher than other studies because of the larger preoperative left atrial diameter of patients enrolled in this study (the average left atrial diameter was $5.2 \mathrm{~cm}$ ) and the large amount of rheumatic valvular disease patients $(52.9 \%)$. We found that increased age $(P=0.002)$ and longer $A F$ duration $(P<0.001)$ were common in AF recurrence group. The expression of the circRNAs showed that there were four circRNAs (circ 81906-RYR2, circ 44782-LAMA2, circ 418-KCNN2 and circ 35880-ANO5) increased significantly in $\mathrm{AF}$ recurrence patients. Furthermore, multivariate analysis showed that increased expression of circ 81906-RYR2 was an independent risk factor for AF recurrence besides advanced age and longer AF duration which were reported in previous study [16, 23]. Nomogram was used to visualize the predictive model and the total score of each patient was calculated by the formula of the predictive model. The results of ROC curve showed that the expression of circ-81906 RYR2 alone was not accuratly enough to predict AF recurrence. It was more effective to make the prediction of $A F$ recurrence by the predictive model. The Kaplan-Meier plot also proved that patients with total score over 70 points was high risk to experience AF recurrence during follow-up, in the other hand, patients under 70 points could be considered as low risk of AF recurrence after surgery.

Themistoclakis el al. reported that recurrence of atrial tachycardias after 2 months were associated with AF recurrence after the blanking period [8]. In this study, 64 patients were found AF recurrence in 3 months after surgery, and 43 patients (67.2\%) among them remain AF till one year after surgery. We also found that 21 patients in AFR1 group restored sinus rhythm and 12 patients in SRM1 group relapsed into AF one-year after surgery. After calculated the predicted points of the AFR1 patients, there were 28 patients under 70 points, and in those patients, who restored sinus rhythm in one year, there were 16 patients under 70 points. In a word, for those low risk patients (total score less than 70 points) who experienced AF 3 months after surgery, early interventions, such as cardioversion and antiarrhythmic drugs therapy, were effective to help restore sinus rhythm in one year.

Several limitations of this study should be mentioned. First, the patient amount and the follow-up length of this study is limited, more AF patients with VHD and longer length of follow-up are needed to make the validation of the predictive model. Second, LAA tissue is necessary for the detection of circ 81906-RYR2, therefore, such kind of detection is restricted to surgical ablation. For those patients received catheter ablation, extra LAA section is needed to get the LAA tissue. Third, AF recurrence is always asymptomatic and undetectable, long term heart rhythm monitor is recommended. 


\section{Conclusion}

Left atrial appendage circular RNA circ 81906-RYR2 was an independent risk factor for AF recurrence after surgical ablation. A predictive model which consist of age, AF duration and circ 81906-RYR2 could be alternative for prediction of AF recurrence. What's more, early intervention for those low recurrent risk patients is helpful to maintain sinus rhythm during follow up.

\section{Abbreviations}

SR: sinus rhythm; AF: atrial fibrillation; VHD: valvular heart disease; circRNA: circular RNA; TTE: transthoracic echocardiography; LAD: left atrial diameter; LVEF: left ventricular ejection fraction; SRM: sinus rhythm maintenance; AFR: atrial fibrillation recurrence; LAA: left atrial appendage; CPB: cardiopulmonary bypass; qRT-PCR: quantitative real-time reverse transcription polymerase chain reaction; SD: standard deviation; ROC: Receiver operating characteristic; AUC: area under the curve.

\section{Declarations}

\section{Ethics approval and consent to participate:}

This study was approved by the ethics committee of the Nanjing Drum Tower Hospital (approval number: 2016-151-01). Written informed consents was obtained from all patients enrolled in this study.

\section{Consent for publication:}

Not applicable.

\section{Availability of data and materials:}

The datasets generated and analyzed during the current study are not publicly available because a series of studies are related to the current study in our project and need to be continued, but are available from the corresponding authors on reasonable request.

\section{Competing interests:}

The authors declare that they have no competing interests.

\section{Fundings:}

This work was supported by the National Natural Science Foundation of China [81970401 to DW, 81800241 to RM]; and the Jiangsu Provincial Medical Youth Talent [QNRC2016035 to QZ].

\section{Authors' contributions:}


QZ and DW conceived the study design; $\mathrm{HCao}$ and FF finished the surgical procedure; $\mathrm{XZ}$ and $\mathrm{YW}$ performed the laboratory experiments and made the follow-up. HChong collected clinical data. $X Z$ and RM completed the data analysis and interpretation. XZ, YW and RM wrote the manuscript. All authors made the approval of the final manuscript.

\section{Acknowledgements:}

The authors thank Dr. Yuzhou Lu and Dr. Shi Xiong of the Department of Cardiothoracic Surgery, Drum Tower Hospital Affiliated to Nanjing University Medical School for surgical samples collection.

\section{References}

1. Baumgartner $\mathrm{H}$, Falk V, Bax JJ, et al. 2017 ESC/EACTS Guidelines for the management of valvular heart disease. European heart journal 2017, 38(36):2739-2791.

2. Rattka $M$, Pott $A$, Kuhberger $A$, et al. Restoration of sinus rhythm by pulmonary vein isolation improves heart failure with preserved ejection fraction in atrial fibrillation patients. Europace 2020, 22(9):1328-1336.

3. Fayad G, Le Tourneau T, Modine T, et al. Endocardial radiofrequency ablation during mitral valve surgery: effect on cardiac rhythm, atrial size, and function. The Annals of thoracic surgery 2005 , 79(5):1505-1511.

4. Geidel S, Ostermeyer J, Lass M, et al. Permanent atrial fibrillation ablation surgery in CABG and aortic valve patients is at least as effective as in mitral valve disease. Thorac Cardiovasc Surg 2006, 54(2):91-95.

5. Gammie JS, Haddad M, Milford-Beland S, et al. Atrial fibrillation correction surgery: lessons from the Society of Thoracic Surgeons National Cardiac Database. The Annals of thoracic surgery 2008, 85(3):909-914.

6. Gillinov AM, Bakaeen F, McCarthy PM, et al. Surgery for paroxysmal atrial fibrillation in the setting of mitral valve disease: a role for pulmonary vein isolation? The Annals of thoracic surgery 2006, 81(1):19-26; discussion 27-18.

7. Pallisgaard JL, Gislason $\mathrm{GH}$, Hansen J, et al. Temporal trends in atrial fibrillation recurrence rates after ablation between 2005 and 2014: a nationwide Danish cohort study. European heart journal 2018, 39(6):442-449.

8. Themistoclakis S, Schweikert RA, Saliba WI, et al. Clinical predictors and relationship between early and late atrial tachyarrhythmias after pulmonary vein antrum isolation. Heart Rhythm 2008, 5(5):679-685.

9. Chen X, Dong JZ, Du X, et al. Long-term outcome of catheter ablation for atrial fibrillation in patients with apical hypertrophic cardiomyopathy. J Cardiovasc Electrophysiol 2018, 29(7):951-957.

10. Nigro JM, Cho KR, Fearon ER, et al. Scrambled exons. Cell 1991, 64(3):607-613. 
11. Westholm JO, Miura P, Olson S, et al. Genome-wide analysis of drosophila circular RNAs reveals their structural and sequence properties and age-dependent neural accumulation. Cell Rep 2014, 9(5):1966-1980.

12. Han B, Chao J, Yao H. Circular RNA and its mechanisms in disease: From the bench to the clinic. Pharmacology \& therapeutics 2018, 187:31-44.

13. Zhu X, Tang X, Chong H, et al. Expression Profiles of Circular RNA in Human Atrial Fibrillation With Valvular Heart Diseases. Front Cardiovasc Med 2020, 7:597932.

14. Robertson JO, Saint LL, Leidenfrost JE, et al. Illustrated techniques for performing the Cox-Maze IV procedure through a right mini-thoracotomy. Annals of cardiothoracic surgery 2014, 3(1):105-116.

15. Kong MH, Lopes RD, Piccini JP, et al. Surgical Maze procedure as a treatment for atrial fibrillation: a meta-analysis of randomized controlled trials. Cardiovasc Ther 2010, 28(5):311-326.

16. Zhao $L$, Jiang $W$, Zhou $L$, et al. The role of valvular regurgitation in catheter ablation outcomes of patients with long-standing persistent atrial fibrillation. Europace 2014, 16(6):848-854.

17. Mulukutla S, Althouse AD, Jain SK, et al. Increased left atrial size is associated with higher atrial fibrillation recurrence in patients treated with antiarrhythmic medications. Clinical cardiology 2018, 41(6):825-829.

18. Jeck WR, Sharpless NE. Detecting and characterizing circular RNAs. Nat Biotechnol 2014, 32(5):453461.

19. Jeck WR, Sorrentino JA, Wang K, et al. Circular RNAs are abundant, conserved, and associated with ALU repeats. RNA 2013, 19(2):141-157.

20. Memczak S, Jens M, Elefsinioti A, et al. Circular RNAs are a large class of animal RNAs with regulatory potency. Nature 2013, 495(7441):333-338.

21. Ashwal-Fluss R, Meyer M, Pamudurti NR, et al. circRNA biogenesis competes with pre-mRNA splicing. Mol Cell 2014, 56(1):55-66.

22. Abe N, Matsumoto K, Nishihara M, et al. Rolling Circle Translation of Circular RNA in Living Human Cells. Sci Rep 2015, 5:16435.

23. Iwamura T, Kajimoto K, Yamamoto T, et al. Mid-term results for the Maze procedure in patients with non-mitral valvular atrial fibrillation. Ann Thorac Cardiovasc Surg 2011, 17(4):356-362.

\section{Figures}


a

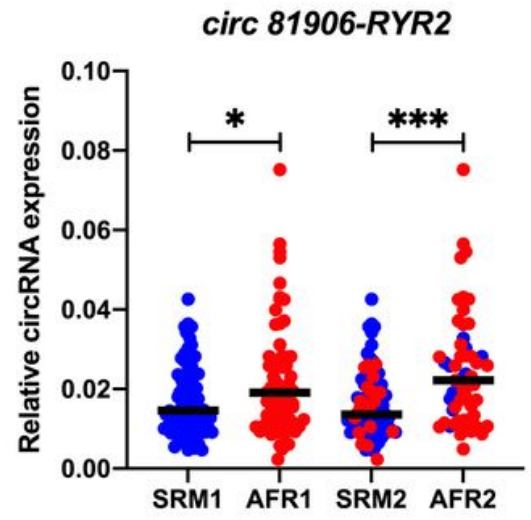

d

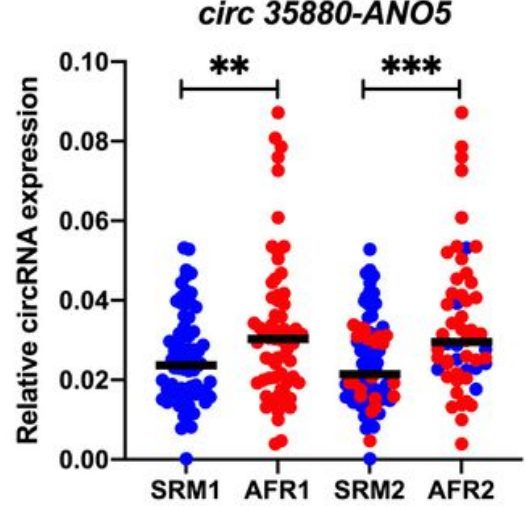

b

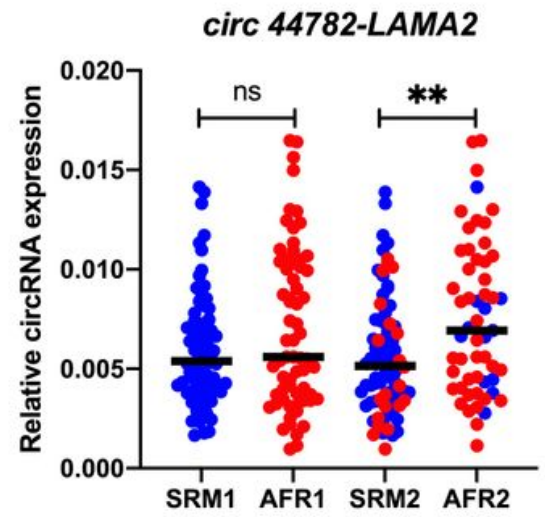

e

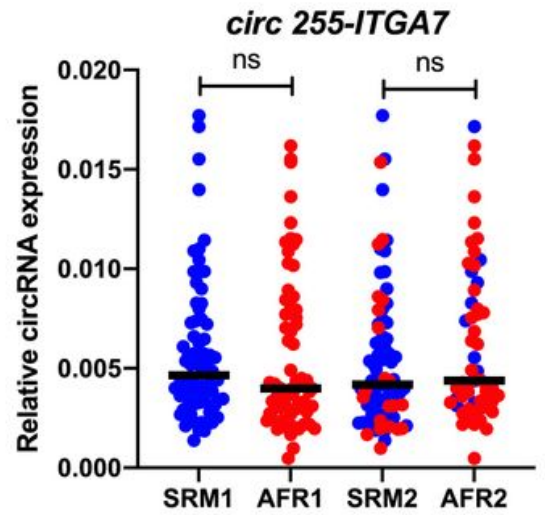

C

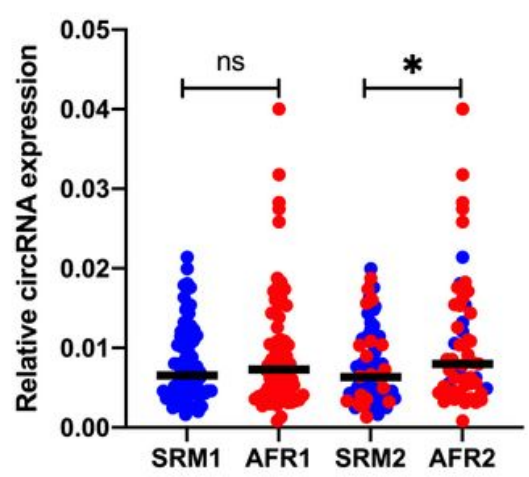

Figure 1

Relative expression of circRNAs in left atrial appendage in valvular PeAF patiens. a. The relative expression of circ 81906-RYR2 was increased in both AFR1 $(P=0.013)$ and AFR2 group $(P<0.001)$. b. The relative expression of circ 44782-LAMA2 was increased in AFR2 group $(P=0.002)$. C. The relative expression of circ 418-KCNN2 was increased in AFR2 group $(P=0.011)$. $d$. The relative expression of circ 35880-AN05 was increased in both AFR1 $(P=0.009)$ and AFR2 group $(P<0.001)$. e. The relative expression of circ 255-ITGA7 was not significantly different between both groups. Blue dots mean patients in SRM1 group and red dots mean patients in AFR1 group. ${ }^{*} \mathrm{P}<0.05{ }^{*} \mathrm{P}<0.01{ }^{*} * \star \mathrm{P}<0.001$ Abbreviations: AFR: atrial fibrillation recurrence; SRM: sinus rhythm maintainess. 


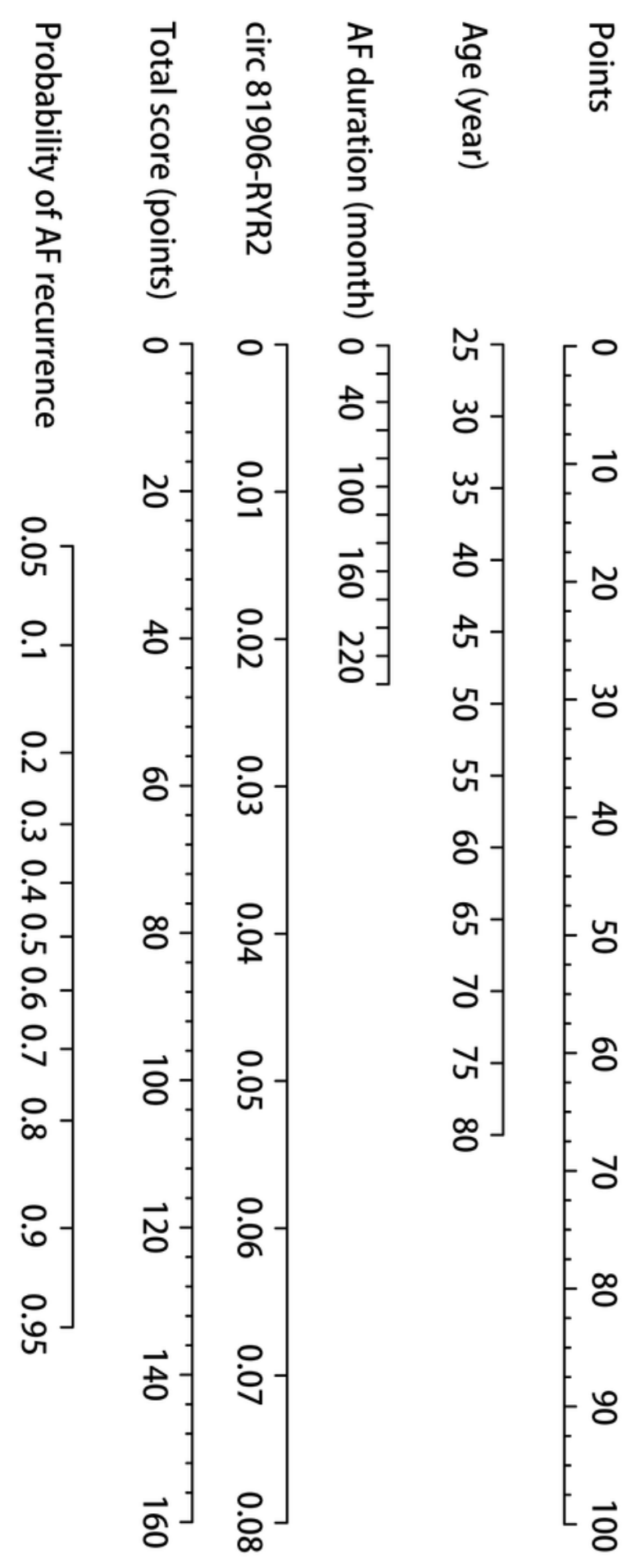

Figure 2

Nomogram of independent risk factors of atrial fibrillation recurrence after surgical ablation. Each value of risk factor could be converted into a detail point and the total score would reflect the probability of atrial fibrillation recurrence in one-year follow up. Abbreviations: AF: atrial fibrillation. 
a

ROC curve

$$
\text { circ 81906-RYR2 }
$$

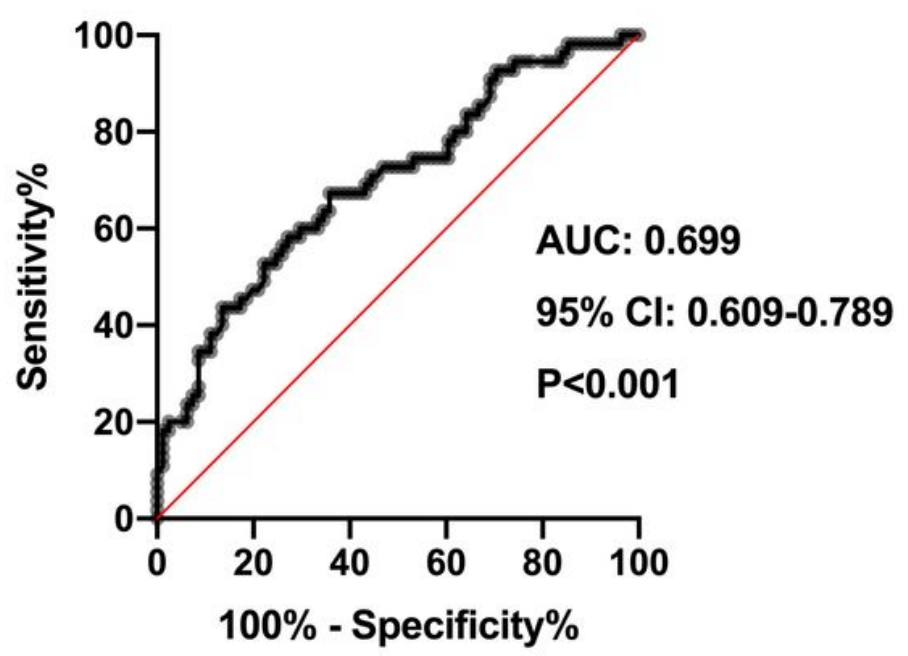

b ROC curve
circ 81906-RYR2 \& Age \& AF duration

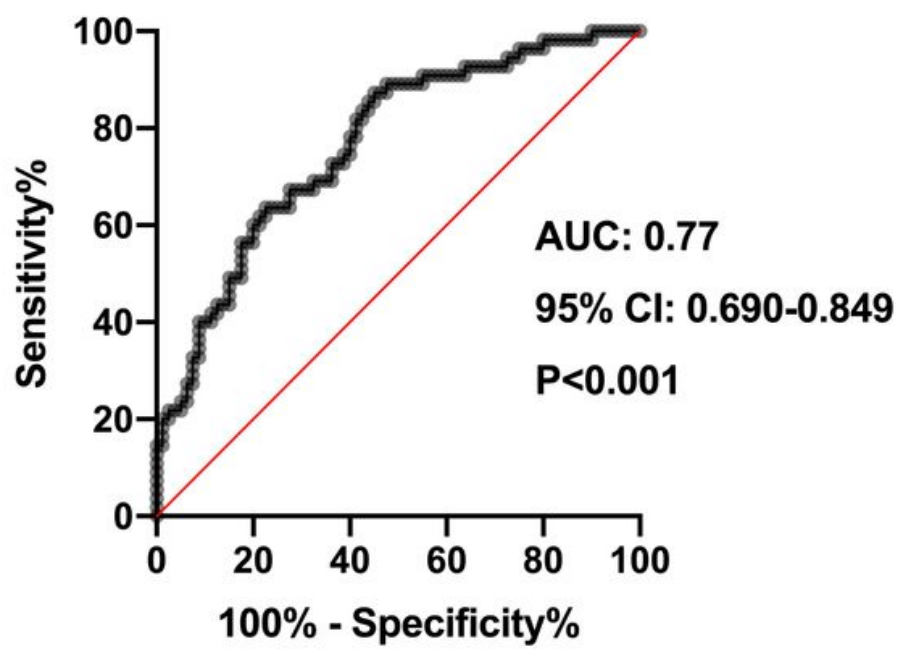

Figure 3

ROC curve of risk factors for predicting AF recurrence after surgical ablation. a. ROC curve of circ 81906RYR2. b. ROC curve of circ 81906-RYR2 combined with age and AF duration. Abbreviations: AF: atrial fibrillation; ROC: receiver operating characteristic. 


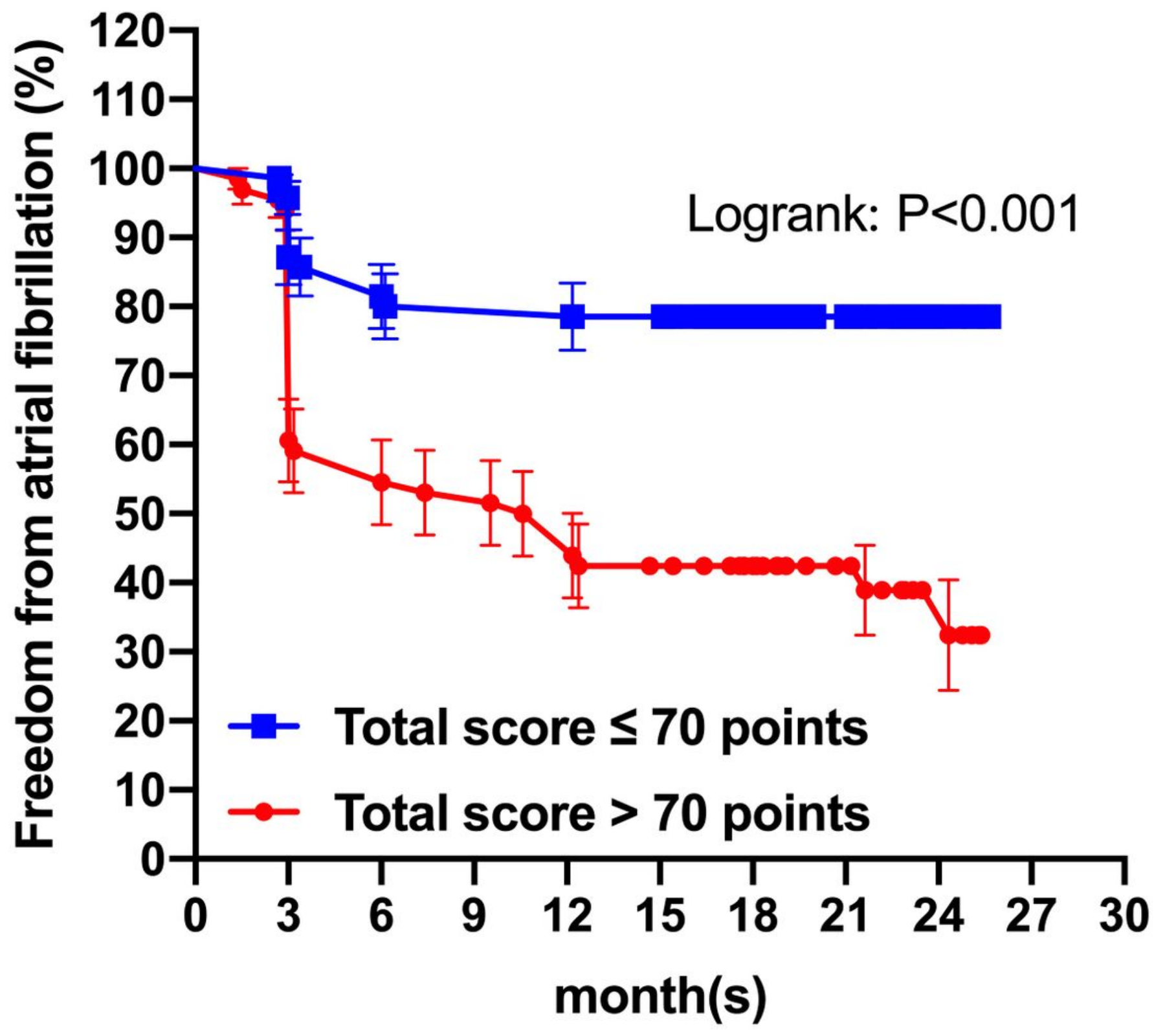

Figure 4

Kaplan-Meier plots of freedom from atrial fibrillation recurrence according to the predictive model.

Patients with total score less than 70 points showed high rate of freedom from AF recurrence in one-year follow up ( $80 \%$ vs $50 \%, P<0.001)$.

\section{Supplementary Files}

This is a list of supplementary files associated with this preprint. Click to download.

- SupplementaryTable1.docx 